Supplementary Information

\title{
Fluid-Assisted Sorted Assembly of Graphene on Polymer
}

\author{
Long Zhu ${ }^{a, b}$, Ji Lang ${ }^{a, c}$, Dong Zhou ${ }^{a, b}$, Qianhong $W u^{a, c^{*}}, B o L i^{a, b^{*}}$ \\ ${ }^{a}$ Department of Mechanical Engineering, Villanova University, Villanova, PA 19085, USA. \\ ${ }^{\mathrm{b}}$ Hybrid Nano-Architectures and Advanced Manufacturing Laboratory, Villanova University, \\ Villanova, PA 19085, USA \\ ${ }^{\mathrm{c}}$ Cellular Biomechanics and Sports Science Laboratory, Villanova University, Villanova, PA \\ 19085, USA
}

No. of pages: 13

No. of figures: 8

No. of tables: 1 
a

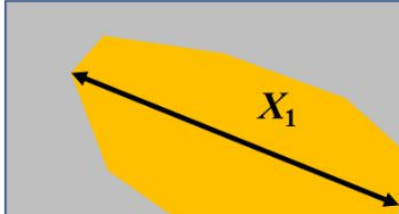

c

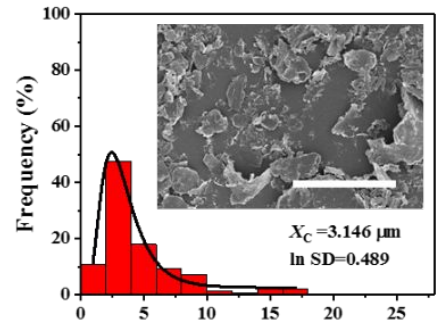

Size $(\mu \mathrm{m})$

f
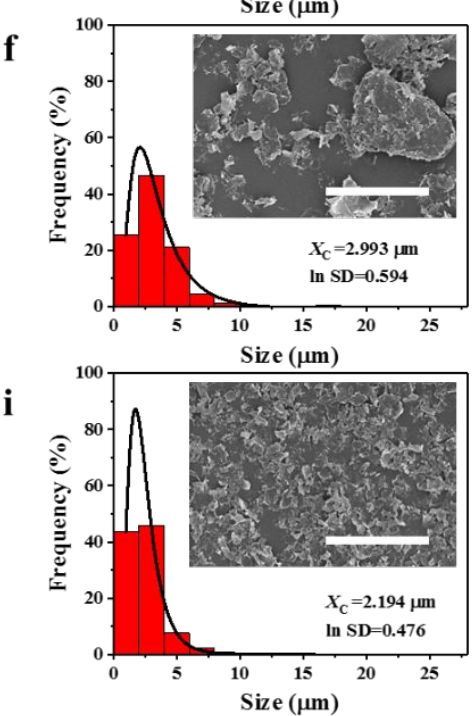

d

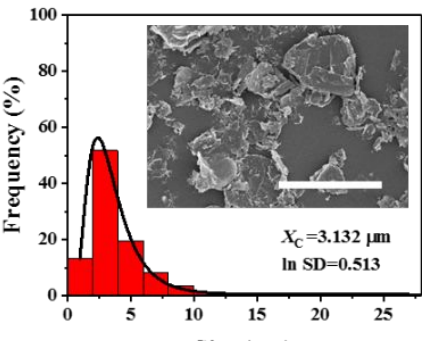

Size $(\mu \mathrm{m})$
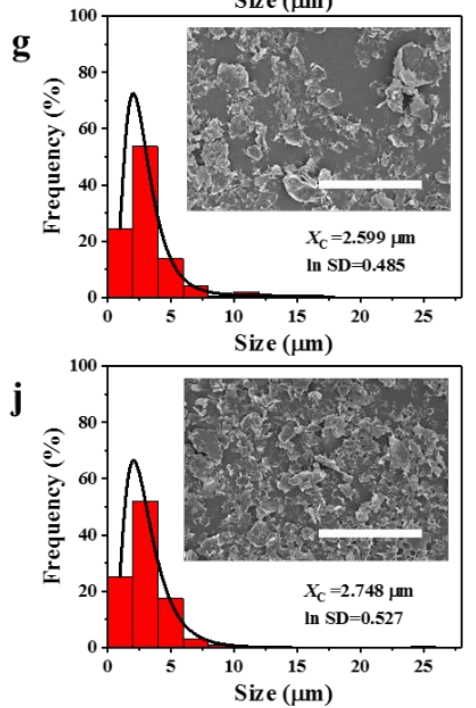

b
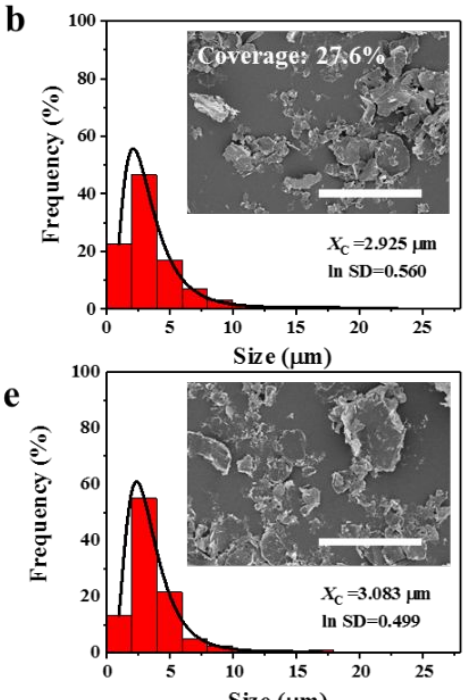

h

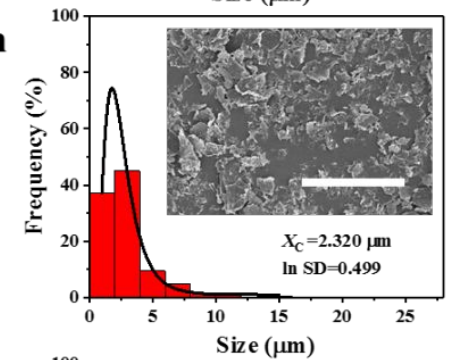

k

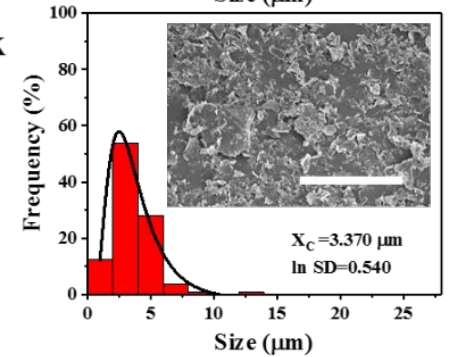

Figure S1. Histogram of lateral size for static assembly and sorted assembly. (a) Schematics of lateral size measurement of the graphene flakes where the largest lateral dimension is chosen. (b) Histogram of lateral size for static assembly. (c-k) Histogram of lateral size for sorted assembly with $h=1 \mathrm{~mm}, \omega=2000 \mathrm{rpm}$, and $t=5 \mathrm{~min}: r=0 \mathrm{~mm}$ (c), $5 \mathrm{~mm}$ (d), $10 \mathrm{~mm}$ (e), $15 \mathrm{~mm}(\mathrm{f}), 20$ $\mathrm{mm}(\mathrm{g}), 25 \mathrm{~mm}(\mathrm{~h}), 30 \mathrm{~mm}(\mathrm{i}), 35 \mathrm{~mm}(\mathrm{j}), 40 \mathrm{~mm}(\mathrm{k})$. Insets: SEM images of the corresponding 
positions. Log-normal fitting is used to determine the average lateral size, $X_{\mathrm{c}}$. The scale bars are $50 \mu \mathrm{m}$.

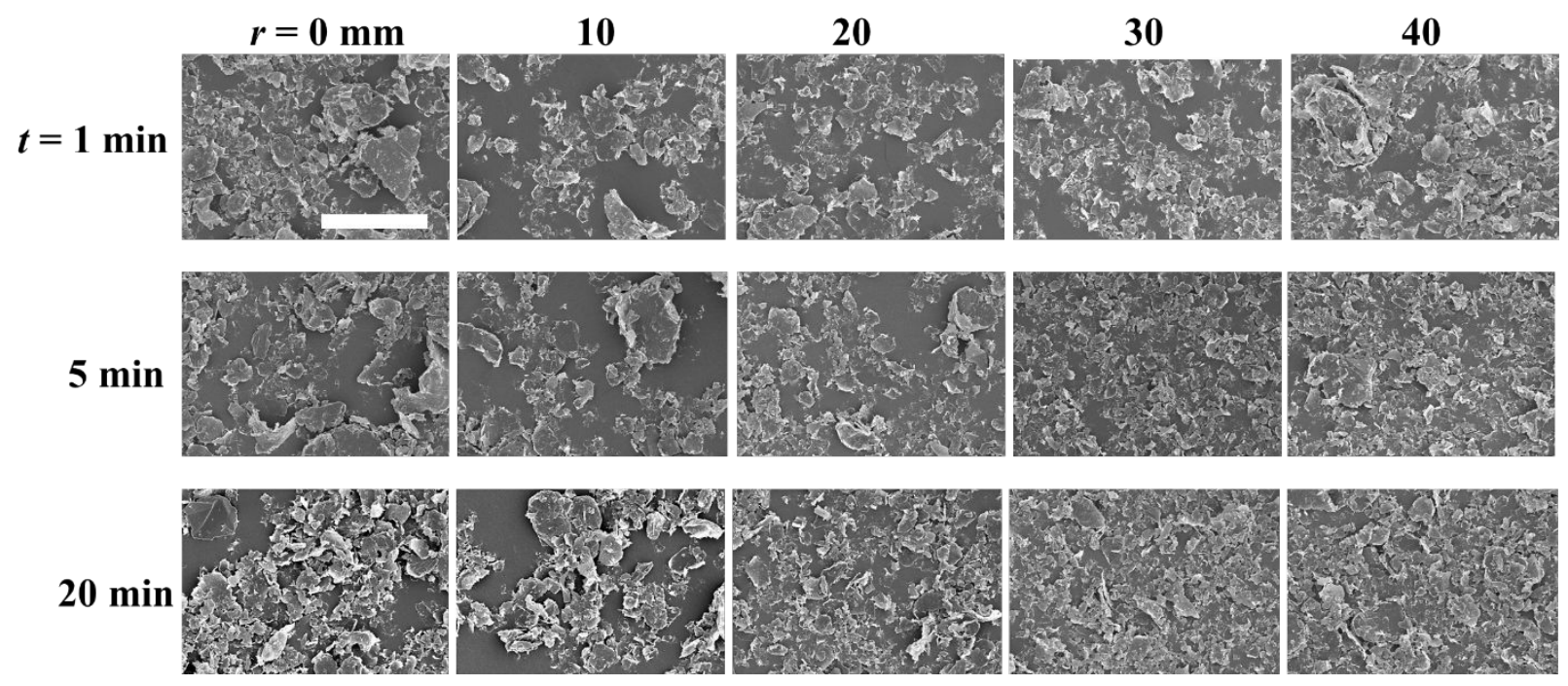

Figure S2. SEM images of assembled graphene at different locations with respect to different assembly times. The gap $(h)$ is $6.5 \mathrm{~mm}$ and rotating speed $(\omega)$ is $2000 \mathrm{rpm}$. Scale bars: $50 \mu \mathrm{m}$ for all images. 

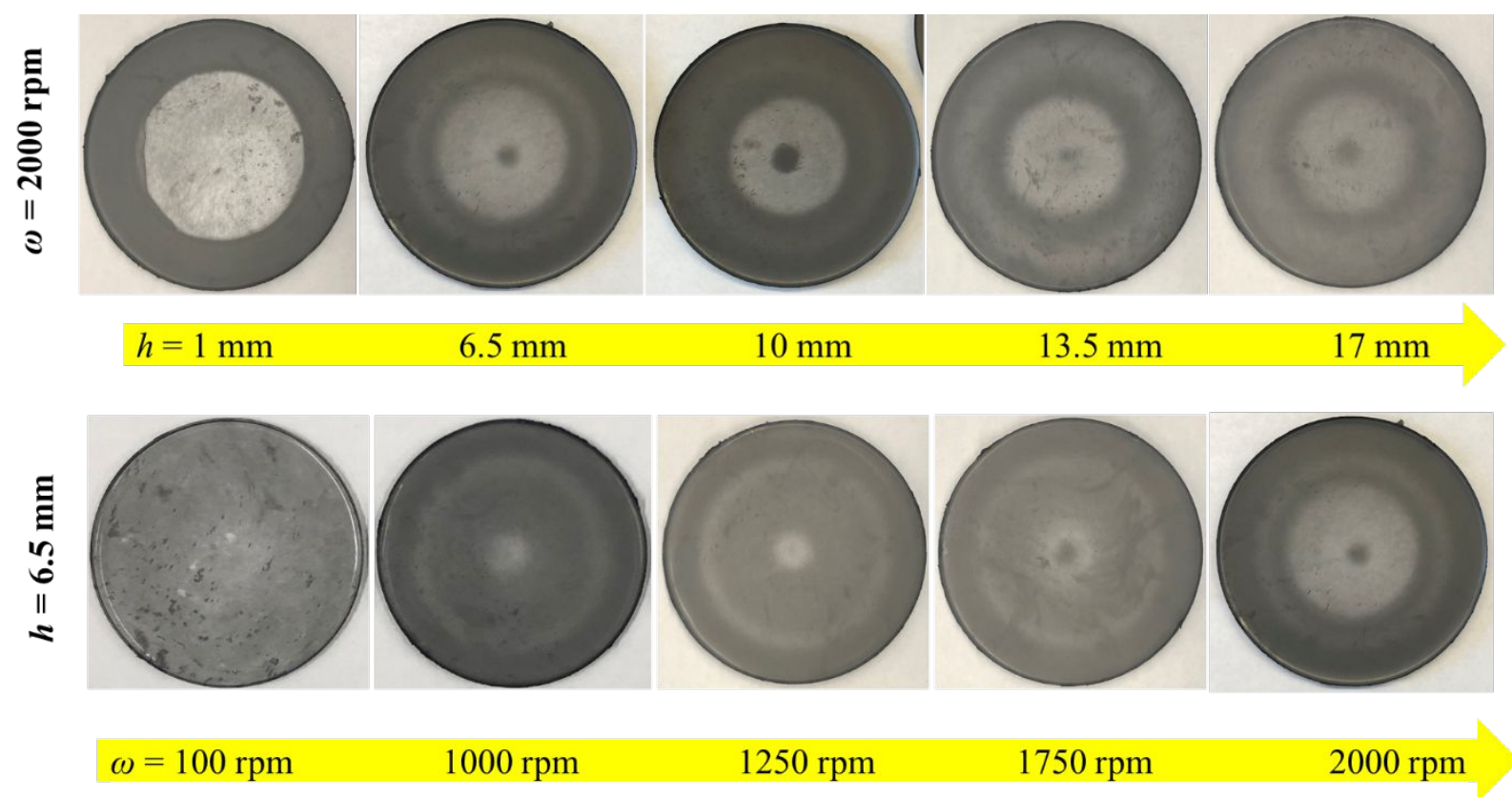

Figure S3. Digital images of assembled graphene-PDMS substrate at different assembly conditions. First row: fix the rotating speed $(\omega=2000 \mathrm{rpm})$ and change the gap $(h=1$ to $17 \mathrm{~mm})$. Second row: fix the gap $(h=6.5 \mathrm{~mm})$ and change the rotating speed ( $\omega=100$ to $2000 \mathrm{rpm}) . R_{\text {PDMS }}$ $=40 \mathrm{~mm}$. 


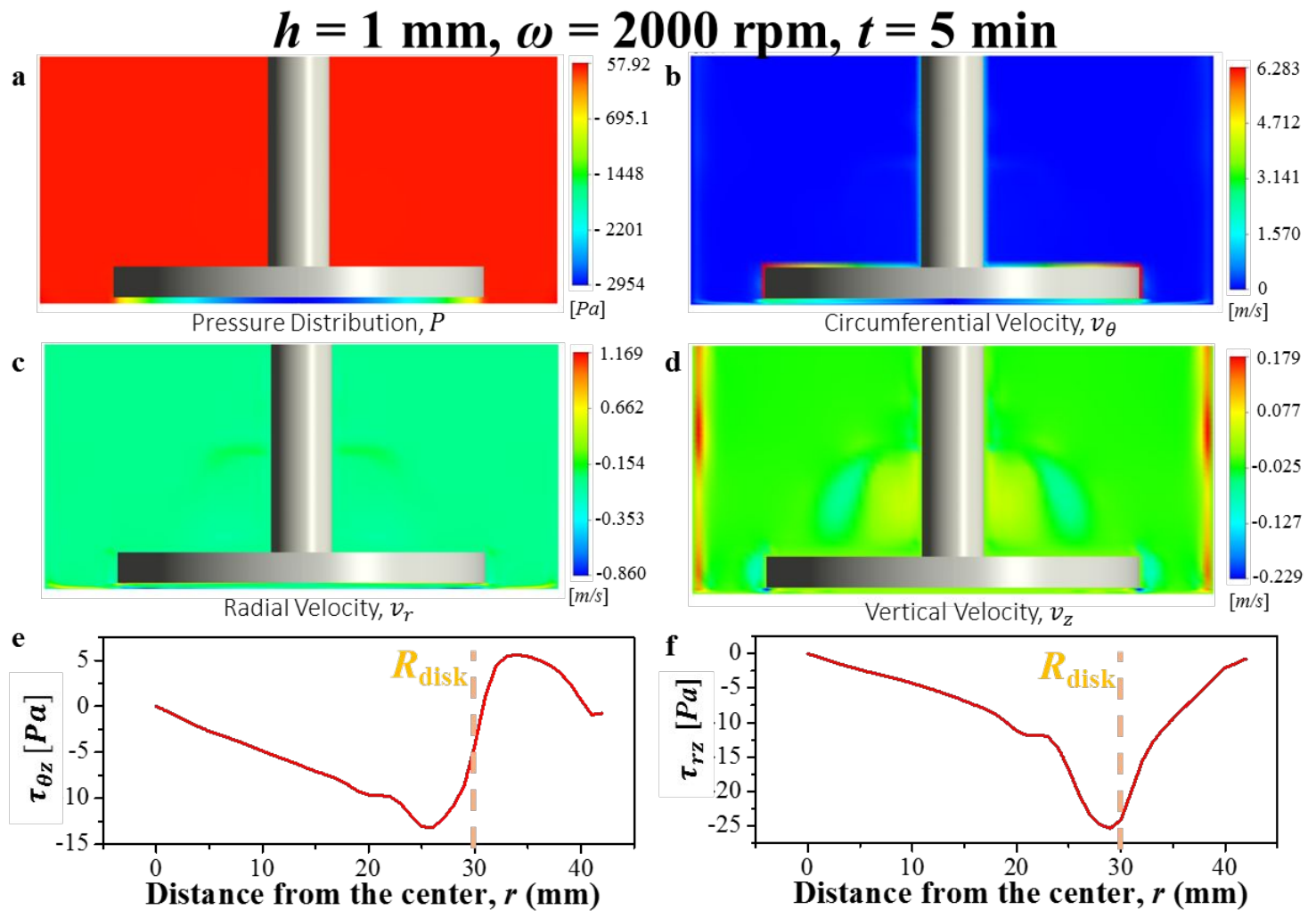

Figure S4. Computational fluid dynamics simulation for the fluid flow under $h=1 \mathrm{~mm}, \omega=2000$ $\mathrm{rpm}$, and $t=5 \mathrm{~min}$, which shows (a) the pressure contour, $P$, (b) circumferential velocity contour, $v_{\theta}$, (c) radial velocity contour, $v_{r}$, (d) vertical velocity contour, $v_{z}$, (e) the distribution of circumferential shear stress on the substrate, $\tau_{\theta z}$ and (f) the distribution of radial shear stress on the substrate, $\tau_{r z}$. 


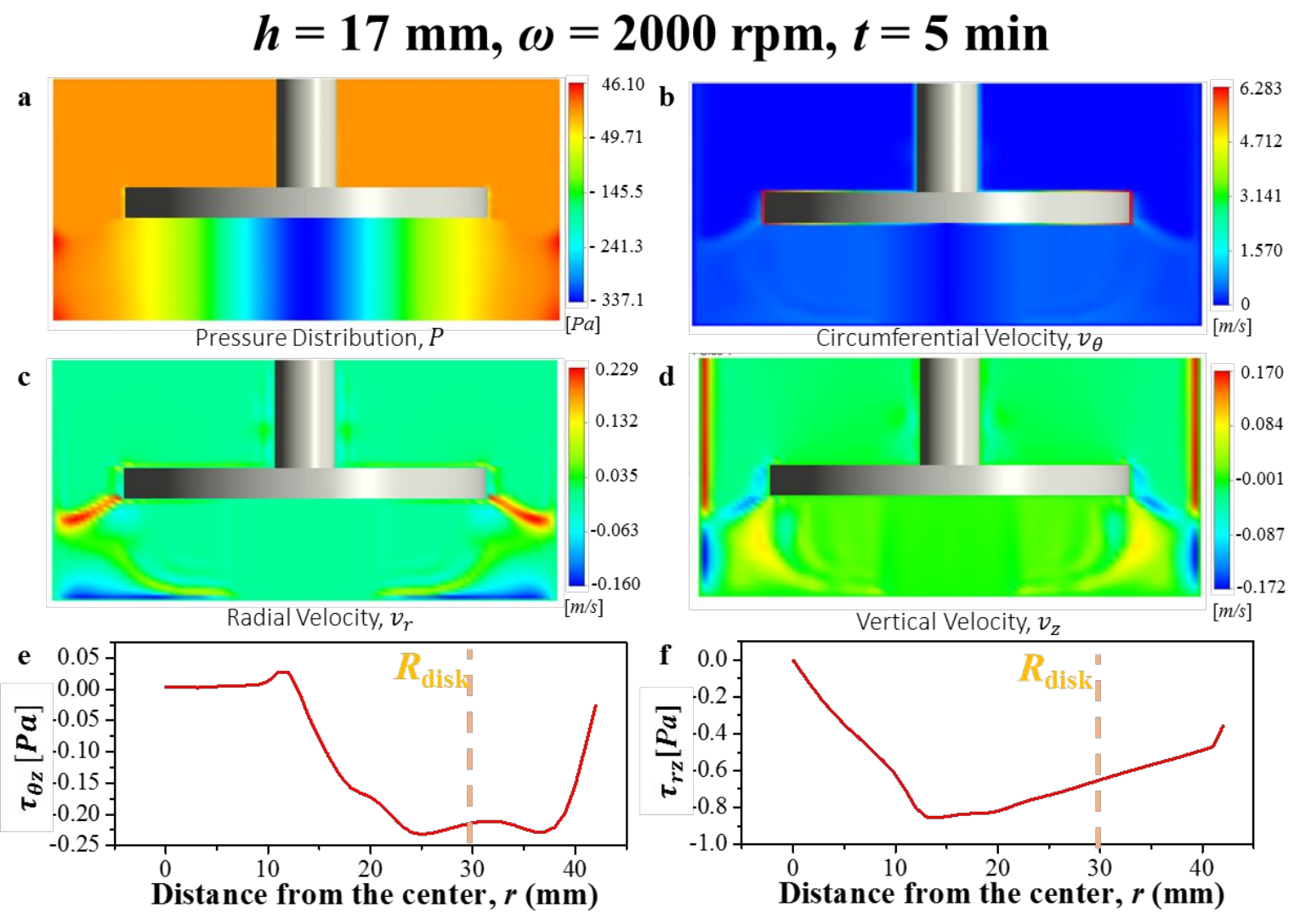

Figure S5. Computational fluid dynamics simulation for the fluid flow under $h=17 \mathrm{~mm}, \omega=$ $2000 \mathrm{rpm}$, and $t=5 \mathrm{~min}$, which shows (a) the pressure contour, $P$, (b) circumferential velocity contour, $v_{\theta}$, (c) radial velocity contour, $v_{r},(\mathrm{~d})$ vertical velocity contour, $v_{z}$, (e) the distribution of circumferential shear stress on the substrate, $\tau_{\theta z}$ and (f) the distribution of radial shear stress on the substrate, $\tau_{r z}$. 


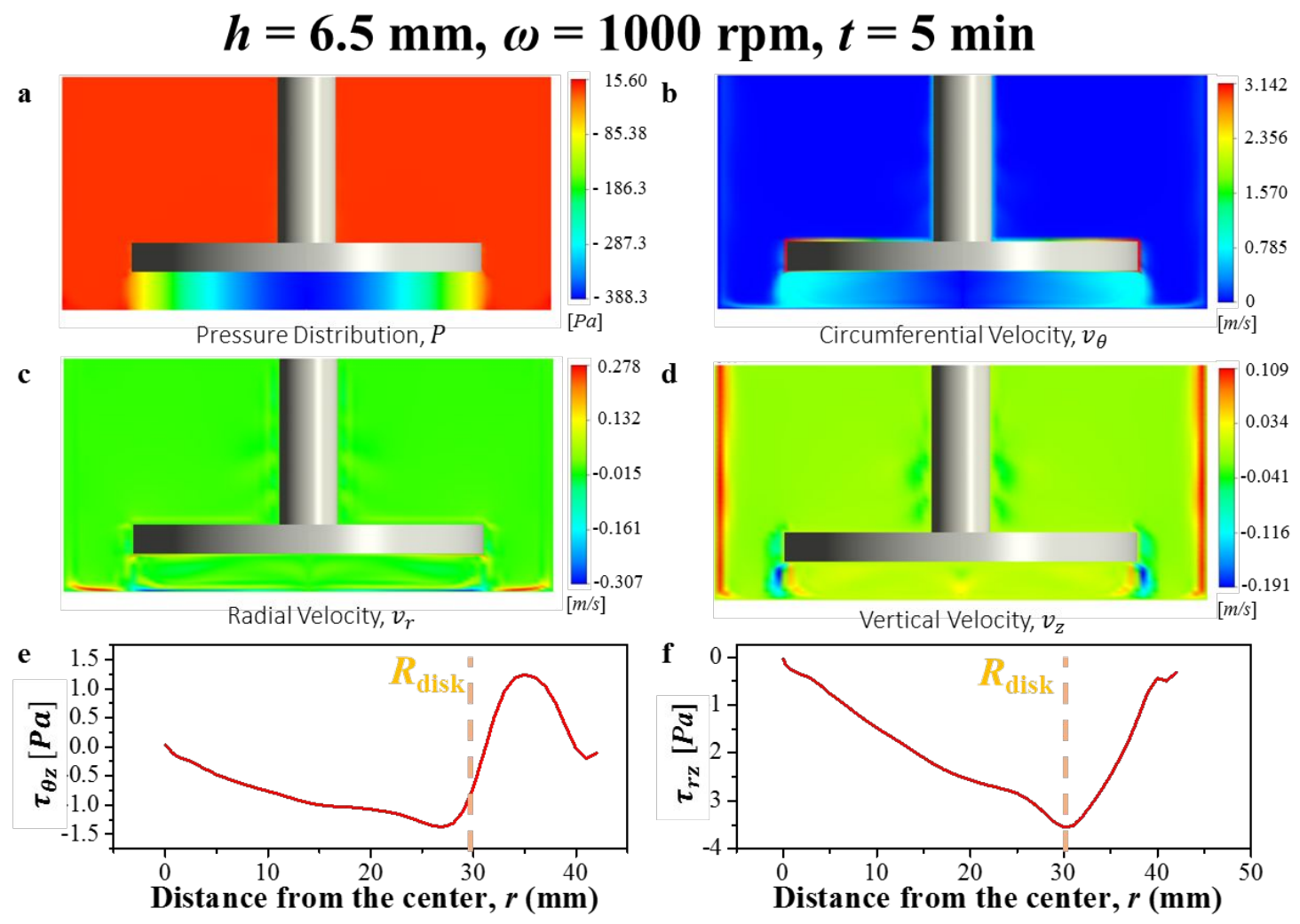

Figure S6. Computational fluid dynamics simulation for the fluid flow under $h=6.5 \mathrm{~mm}, \omega=$ $1000 \mathrm{rpm}$, and $t=5 \mathrm{~min}$, which shows (a) the pressure contour, $P$, (b) circumferential velocity contour, $v_{\theta}$, (c) radial velocity contour, $v_{r}$, (d) vertical velocity contour, $v_{z}$, (e) the distribution of circumferential shear stress on the substrate, $\tau_{\theta z}$ and (f) the distribution of radial shear stress on the substrate, $\tau_{r z}$. 


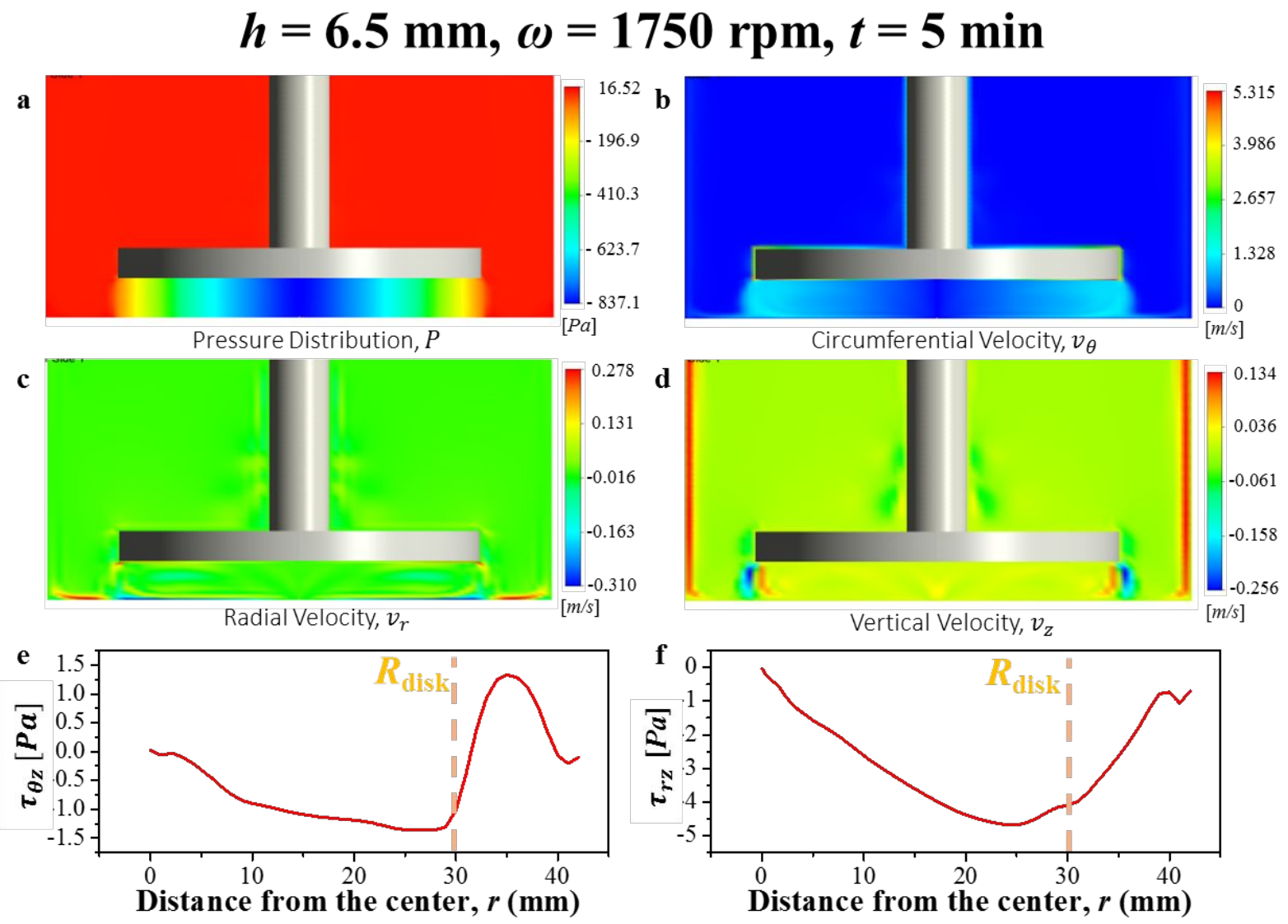

Figure S7. Computational fluid dynamics simulation for the fluid flow under $h=6.5 \mathrm{~mm}, \omega=$ $1750 \mathrm{rpm}$, and $t=5 \mathrm{~min}$, which shows (a) the pressure contour, $P$, (b) circumferential velocity contour, $v_{\theta}$, (c) radial velocity contour, $v_{r},(\mathrm{~d})$ vertical velocity contour, $v_{z}$, (e) the distribution of circumferential shear stress on the substrate, $\tau_{\theta z}$ and (f) the distribution of radial shear stress on the substrate, $\tau_{r z}$. 

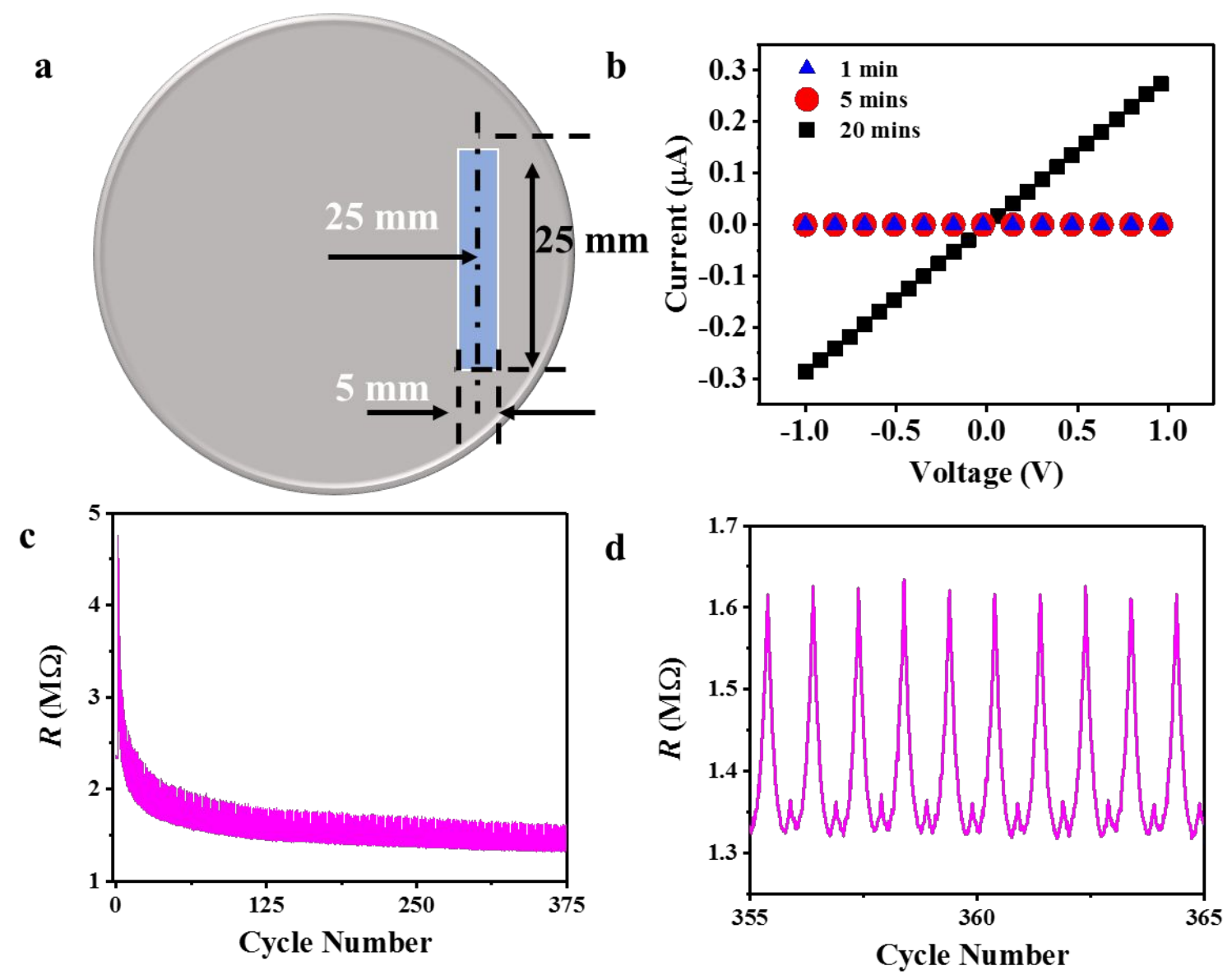

Figure S8. (a) The schematics of sample preparation for strain sensor. (b) I-V curves of samples with different assembly time, and the 20 min sample was chosen for sensing test. (c) and (d) The training process of sensor include 375 stretch-release cycles at $1 \%$ strain. 
Table S1 The performance comparison of graphene-based strain sensors.

\begin{tabular}{|c|c|c|c|c|}
\hline Material & $\begin{array}{l}\text { Maximum } \\
\text { sensing range }\end{array}$ & $\begin{array}{l}\text { Detection } \\
\text { limit }\end{array}$ & Gauge factor & Ref. \\
\hline Graphene on PDMS & $5 \%$ & $0.02 \%$ & 70.1 & This work \\
\hline Few-layer graphene on PDMS & $7.1 \%$ & $0.015 \%$ & $\begin{array}{l}2.4 \text { at strains }< \\
1.8 \%, 4-14 \text { at } \\
\text { strains }>1.8 \%\end{array}$ & 1 \\
\hline $\begin{array}{l}\mathrm{MoS}_{2} \text {-decorated laser-induced } \\
\text { graphene }\end{array}$ & $37.5 \%$ & $0.025 \%$ & 1242 & 2 \\
\hline Single-layer graphene & $20 \%$ & $0.1 \%$ & 42.2 & 3 \\
\hline Fish scale-like rGO/tape film & $82 \%$ & $0.1 \%$ & $16.2,150$ & 4 \\
\hline $\begin{array}{l}\text { Graphene-coated springlike } \\
\text { mesh network }\end{array}$ & $110 \%$ & $0.1 \%$ & 1.63 at $>10 \%$ & 5 \\
\hline Fabricated by pencil drawn & $0.6 \%$ & $0.13 \%$ & 536.6 & 6 \\
\hline Graphene nanosheets & $87.5 \%$ & $0.2 \%$ & 45573.1 & 7 \\
\hline Nanographene films on PDMS & $1.6 \%$ & $0.2 \%$ & 500 & 8 \\
\hline $\begin{array}{l}\text { Fiber of compression spring } \\
\text { architecture }\end{array}$ & $100 \%$ & $0.2 \%$ & 3.7 & 9 \\
\hline $\begin{array}{l}\text { Graphene micro-ribbon strain } \\
\text { sensor }\end{array}$ & $10 \%$ & $0.5 \%$ & 9.49 & 10 \\
\hline $\begin{array}{l}\text { Graphene composite films } \\
\text { with layered structure }\end{array}$ & $25 \%$ & $0.5 \%$ & $6-35$ & 11 \\
\hline $\begin{array}{l}\text { Graphene mesh fabric on } \\
\text { stretchable tape }\end{array}$ & $7.5 \%$ & $1 \%$ & $\begin{array}{l}20 \text { at strains } \\
<5 \% \text { and } \\
\text { around } 1000 \text { at } \\
\text { strains }>7.5 \%\end{array}$ & 12 \\
\hline Fragmentized graphene foam & $77 \%$ & $\begin{array}{l}\text { Less than } 1 \\
\%\end{array}$ & 29 & 13 \\
\hline
\end{tabular}




\begin{tabular}{|c|c|c|c|c|}
\hline $\begin{array}{l}\text { Nitrogen-doped graphene } \\
\text { sponge }\end{array}$ & $20 \%$ & $2 \%$ & N/A & 14 \\
\hline $\begin{array}{l}\text { Graphene/ionic } \\
\text { conductor-Ecoflex }\end{array}$ & $300 \%$ & $4 \%$ & 25.2 & 15 \\
\hline Graphene-nanocellulose paper & $100 \%$ & $10 \%$ & 7.1 & 16 \\
\hline $\begin{array}{l}\text { Reduced graphene oxide } \\
\text { (RGO)-decorated flexible } \\
\text { thermoplastic polyurethane } \\
\text { (TPU) electrospun fibrous } \\
\text { mats }\end{array}$ & $100 \%$ & $10 \%$ & $\begin{array}{l}11 \text { at } 10 \% \text { and } \\
79 \text { at } 100 \%\end{array}$ & 17 \\
\hline $\begin{array}{l}\mathrm{SiO}_{2} / \text { graphene- } \\
\text { decoratedelectrospunnanofiber }\end{array}$ & $110 \%$ & $10 \%$ & 5.9 & 18 \\
\hline
\end{tabular}

\section{REFERENCES}

(1) Bae, S.-H.; Lee, Y.; Sharma, B. K.; Lee, H.-J.; Kim, J.-H.; Ahn, J.-H. Graphene-based transparent strain sensor. Carbon 2013, 51, 236-242.

(2) Chhetry, A.; Sharifuzzaman, M.; Yoon, H.; Sharma, S.; Xuan, X.; Park, J. Y. $\mathrm{MoS}_{2^{-}}$ Decorated Laser-Induced Graphene for a Highly Sensitive, Hysteresis-free, and Reliable Piezoresistive Strain Sensor. ACS Appl. Mater. Interfaces 2019, 11, 22531-22542.

(3) Chun, S.; Choi, Y.; Park, W. All-graphene strain sensor on soft substrate. Carbon 2017, 116, 753-759.

(4) Liu, Q.; Chen, J.; Li, Y.; Shi, G. High-performance strain sensors with fish-scale-like graphene-sensing layers for full-range detection of human motions. ACS Nano 2016, 10, 79017906.

(5) Yuan, W.; Yang, J.; Yang, K.; Peng, H.; Yin, F. High-performance and multifunctional skinlike strain sensors based on graphene/springlike mesh network. ACS Appl. Mater. Interfaces 2018, 10, 19906-19913. 
(6) Liao, X.; Liao, Q.; Yan, X.; Liang, Q.; Si, H.; Li, M.; Wu, H.; Cao, S.; Zhang, Y. Flexible and highly sensitive strain sensors fabricated by pencil drawn for wearable monitor. Adv. Funct. Mater. 2015, 25, 2395-2401.

(7) Tang, Y.; Guo, Q.; Chen, Z.; Zhang, X.; Lu, C.; Cao, J.; Zheng, Z. Scalable Manufactured Self-Healing Strain Sensors Based on Ion-Intercalated Graphene Nanosheets and Interfacial Coordination. ACS Appl. Mater. Interfaces 2019, 11, 23527-23534.

(8) Zhao, J.; Wang, G.; Yang, R.; Lu, X.; Cheng, M.; He, C.; Xie, G.; Meng, J.; Shi, D.; Zhang, G. Tunable piezoresistivity of nanographene films for strain sensing. ACS Nano 2015, 9, 16221629 .

(9) Cheng, Y.; Wang, R.; Sun, J.; Gao, L. A stretchable and highly sensitive graphene-based fiber for sensing tensile strain, bending, and torsion. Adv. Mater. 2015, 27, 7365-7371.

(10) Tian, H.; Shu, Y.; Cui, Y.-L.; Mi, W.-T.; Yang, Y.; Xie, D.; Ren, T.-L. Scalable fabrication of high-performance and flexible graphene strain sensors. Nanoscale 2014, 6, 699-705.

(11) Liu, Y.; Zhang, D.; Wang, K.; Liu, Y.; Shang, Y. A novel strain sensor based on graphene composite films with layered structure. Composites, Part A 2016, 80, 95-103.

(12) Liu, Q.; Zhang, M.; Huang, L.; Li, Y.; Chen, J.; Li, C.; Shi, G. High-quality graphene ribbons prepared from graphene oxide hydrogels and their application for strain sensors. ACS Nano 2015, 9, 12320-12326.

(13) Jeong, Y. R.; Park, H.; Jin, S. W.; Hong, S. Y.; Lee, S. S.; Ha, J. S. Highly stretchable and sensitive strain sensors using fragmentized graphene foam. Adv. Funct. Mater. 2015, 25, 42284236.

(14) Huang, J.; Liu, X.; Yang, Z.; Wu, X.; Wang, J.; Yang, S. Extremely elastic and conductive $\mathrm{N}$-doped graphene sponge for monitoring human motions. Nanoscale 2019, 11, 1159-1168.

(15) Liu, C.; Han, S.; Xu, H.; Wu, J.; Liu, C. Multifunctional Highly Sensitive Multiscale Stretchable Strain Sensor Based on a Graphene/Glycerol-KCl Synergistic Conductive Network. ACS Appl. Mater. Interfaces 2018, 10, 31716-31724. 
(16) Yan, C.; Wang, J.; Kang, W.; Cui, M.; Wang, X.; Foo, C. Y.; Chee, K. J.; Lee, P. S. Highly stretchable piezoresistive graphene-nanocellulose nanopaper for strain sensors. Adv. Mater. 2014, $26,2022-2027$.

(17) Wang, Y.; Hao, J.; Huang, Z.; Zheng, G.; Dai, K.; Liu, C.; Shen, C. Flexible electrically resistive-type strain sensors based on reduced graphene oxide-decorated electrospun polymer fibrous mats for human motion monitoring. Carbon 2018, 126, 360-371.

(18) Gao, J.; Li, B.; Huang, X.; Wang, L.; Lin, L.; Wang, H.; Xue, H. Electrically conductive and fluorine free superhydrophobic strain sensors based on $\mathrm{SiO}_{2} /$ graphene-decorated electrospun nanofibers for human motion monitoring. Chem. Eng. J. 2019, 373, 298-306. 